\title{
Effect of annealing temperature on structural, optical and photocatalytic properties of $\mathrm{CuO}$ nanoparticles
}

\author{
Loubaba. Attou ${ }^{1,3, *}$, Boujemaâ. Jaber ${ }^{2}$ and Hamid. Ez-Zahraouy ${ }^{1}$ \\ ${ }^{1}$ LaMCScI, Department of Physics, Faculty of Sciences, Mohammed V University, Rabat, Morocco \\ ${ }^{2}$ Materials Science Platform, UATRS division, CNRST, Rabat, Morocco \\ ${ }^{3}$ Institute for Materials \& Surface Technology (IMST), HAW Kiel-University of Applied Sciences, Grenzstraße3, \\ D-24149 Kiel, Germany
}

\begin{abstract}
In this study, pure crystallographic $\mathrm{CuO}$ nanoparticles are successfully synthesized via the sol-gel method through the reduction of copper acetate in water as a solvent. The as-prepared nanoparticles are characterized by X-ray diffraction (XRD), UV-Vis spectrophotometer and transmission electron microscopy (TEM). In order to study the effect of annealing temperature on their morphological and optical behavior, the obtained nanoparticles are annealed at different temperatures, from $200^{\circ} \mathrm{C}$ to $800^{\circ} \mathrm{C}$. XRD studies revealed that by increasing temperature, the full width at half maximum (FWHM) of peaks decreases indicating the enhancement of the grain size of the $\mathrm{CuO}$ nanoparticles. Our result also reveals that the optical band gap decreases with the increase of temperature, which can be fully reflected by its variation from $3.83 \mathrm{eV}$ to $2.21 \mathrm{eV}$ when nanoparticle's size varied from $9.84 \mathrm{~nm}$ to $188.33 \mathrm{~nm}$ respectively, figured out in TEM analysis. In the studied annealed range temperature, the obtained $\mathrm{Eg}$ values remain widely higher than reported bulk values. The photocatalytic deactivation/degradation process significantly enhanced the activity of CuO-NPs under UV-visible light in the presence of $\mathrm{MB}$ dye, we compare then the photodegradation spectra of CuO-NPs annealed at $200^{\circ} \mathrm{C}, 400^{\circ} \mathrm{C}, 600^{\circ} \mathrm{C}$ and $800^{\circ} \mathrm{C}$, obtained by UV-visible spectroscopy.
\end{abstract}

Keywords: $\mathrm{CuO}$ nanoparticles; sol-gel; material characterization; quantum confinement.

\section{Introduction}

Recently, researches on nanoparticles and nanostructures have received a great deal of attention not only in the area of synthesis and characterization but also in their potential application in various hightechnological fields. Metal oxide nanoparticles represent a field of this nanomaterials chemistry, which attracts considerable interest due to the potential technological applications of these compounds and display the most fascinating and widest range of properties ${ }^{1}$. Among the transition metal oxides, Copper(II) oxide that reveal narrow band-gap (Eg) of 1.0-2.08 eV, behaves as a p-type semiconductor ${ }^{2}$, this semiconducting nature gives them a real importance in several applications, such as gas sensors, magnetic storage media, solar energy transformation and especially in the field of Photocatalysis ${ }^{3}$. Photocatalytic degradation has been proved as a promising method for the treatment of wastewater, contaminated with organic and inorganic pollutants. The process, as a means of removal of persistent water contaminants such as dyes and pesticides, has attracted the attention of many researchers in recent years ${ }^{4}$.

*Corresponding author: Loubaba Attou

Email address: atloubaba@gmail.com

DOI: http://dx.doi.org/10.13171/mjc751911261230la
The photo-catalytic degradation mechanism as presented in scheme 1 starts with the absorption of photons (with sufficient energy that equals or exceeds the band-gap energy of the catalyst) through light 5; the electron becomes excited and jumps to the conduction band, leaving a positively charged hole in the valence band. Besides recombination with the electron, the positively charged hole can oxidize water molecules to form hyper-reactive hydroxyl free radicals $(\mathrm{OH})^{*}$, the resulting hydroxyl radicals are the main agent that attack the chemical pollutant molecules or microorganism cells to purify water. The excited electron can react with a dissolved oxygen molecule to form oxygen radical, which is also active toward organic pollutants ${ }^{4}$.

There have been many research efforts devoted to improving the photocatalytic activity. An alternative type of catalysts for photodegradation of organic dyes is nanoparticles of some transition metals such as silver, copper and copper oxide (II) since it was reported by Tadjarodi et al. ${ }^{6}$ as having a good photocatalytic behavior when combining it with $\mathrm{H}_{2} \mathrm{O}_{2}$ with a degradation efficiency almost $100 \%$ toward rhodamine B. It was also reported by ${ }^{7}$, that $\mathrm{CuO}$ (II) exhibits an enhanced 
photocatalytic performance when annealing it at higher temperature.

The synthesis procedure plays a crucial role in controlling the size, the shape of the nanostructure and hence detecting different properties of the material. Various methods are used for the synthesis of $\mathrm{CuO}$ nanoparticles including sol-gel ${ }^{8}$, solid-state reaction, microwave irradiation and thermal decomposition. Among all the processes sol-gel method was reported as having many advantageous aspects as pointed out by Chand $\&$ al ${ }^{8}$, such as producing materials at ultra-low temperatures, synthesizing almost any material, cosynthesizing two or more materials simultaneously, controlling the microstructure, the physical, the mechanical and the chemical properties of the final products etc.. ${ }^{9}$.

In the present work, we have reported the synthesis of $\mathrm{CuO}$ NPs via the sol-gel method and the effect of grain size on their structural and optical characterization for photocatalytic application. The photocatalytic activity of the obtained NPs examined using the degradation of methylene blue (MB) under UV and visible light in two different annealing temperature to see how we can control the photodegradation of the material for each temperature.

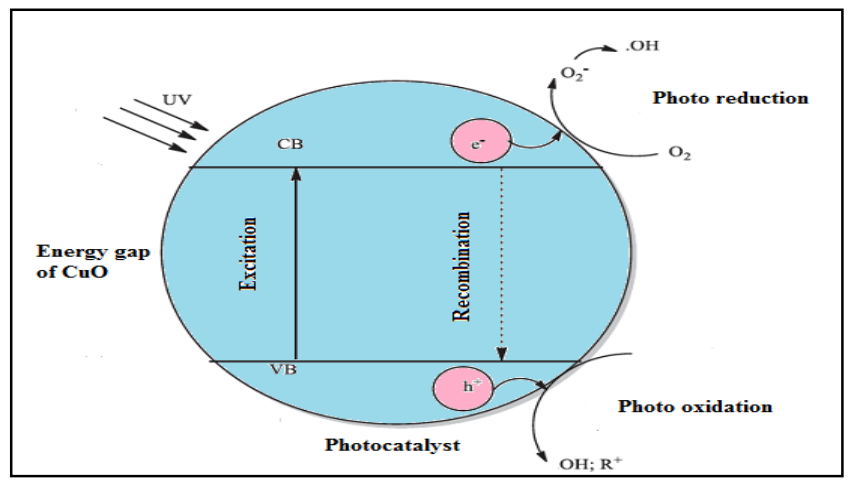

Scheme 1. Illustration of photocatalysis mechanism on $\mathrm{CuO}$

\section{Experimental details}

\section{Chemical Synthesis:}

All chemicals used in this study were analytical grade and used without further purification. We used Deionized water in all experiments. For the synthesis of $\mathrm{CuO}$ nanoparticles in the sol-gel process, $1.98 \mathrm{~g}$ of $(\mathrm{Cu}(\mathrm{CH} 3 \mathrm{COO}) 2, \mathrm{H} 2 \mathrm{O})$ as a precursor were dissolved and stirred for 1 hour in $100 \mathrm{ml}$ of water to get a homogenous solution. Then $0.8 \mathrm{M}$ of $\mathrm{NaOH}$ was added to this solution maintained at $60^{\circ} \mathrm{C}$ under constant stirring during 2 hours. Finally, the obtained black precipitate was centrifuged at $5000 \mathrm{rpm}$ during $40 \mathrm{~min}$, washed twice with distilled water, once with absolute ethanol to remove all kinds of impurities then dried at $50{ }^{\circ} \mathrm{C}$ for 12 hours. In order to evaluate the effect of the temperature on the structural, morphological and optical properties, the samples were annealed under four different temperatures: $200{ }^{\circ} \mathrm{C}, 400^{\circ} \mathrm{C}, 600^{\circ} \mathrm{C}$ and $800^{\circ} \mathrm{C}$ respectively. For all reactions, the copper acetate, $\mathrm{NaOH}$ concentrations, temperature and time of the reaction were maintained constant.

\section{Characterization}

The obtained powders were characterized by X-Ray Diffraction with the use of the Panalytical XPERT-PRO powder diffractometer with the $\mathrm{Cu}-\mathrm{K} \alpha$ radiation. The Rietveld refinement was performed using MAUD (material analysis using diffraction) software. The Rietveld routine was performed in the $2 \theta$ range from $10^{\circ}$ to $90^{\circ}$, using an angular step of $0.03^{\circ} / \mathrm{min}$ and exposure time of $3 \mathrm{~s}$. The morphology of the prepared $\mathrm{CuO}$ nanoparticles was carried out using the FEI Transmission
Electron Microscopy (TEM, Tecnai G2 12 TWIN, $120 \mathrm{KV}$ ) with a LaB6 filament. The UV-Visible absorption spectra were recorded using the UV-Visible Perkin Elmer spectrophotometer (Lambda 900), We added Powders into the water and sonicated in an ethanol bath for $10 \mathrm{~min}$ to form a homogeneous suspension with the concentration of about $0.1 \mathrm{~g} / 5 \mathrm{ml}$.

\section{Photocatalytic activity}

The activity of the annealed $\mathrm{CuO}$ NPs was evaluated using methylene blue (MB) under a UV-source functioning as a lamp of the spherical shape of $125 \mathrm{~W}$ AB-C (200-600 nm) irradiation. A solid sample (200 mg) was preliminary dispersed in $100 \mathrm{~mL}$ of a dye solution (10 $\mathrm{mgL}-1$ ). Before UV irradiation, an adsorption-desorption equilibrium is establishing by stirring during $10 \mathrm{~min}$, based on the kinetics studies shown in (Fig.7), the solution is then, exposed to UV light under magnetic stirring. At each selected time, the suspensions is centrifuging at $14000 \mathrm{rpm}$ for $15 \mathrm{~min}$, and the supernatants are stored in the dark. The concentration of dye remaining in solution $\mathrm{C}\left(\mathrm{mg} . \mathrm{L}^{-1}\right)$ is measuring by using a UV-visible spectrophotometer (PerkinElmer Lambda II).

\section{Results and discussion}

\section{Structural properties}

$\mathrm{X}$-ray diffraction measurements were carried out to study the crystal structure and crystalline quality. (Fig.1) represents the XRD patterns of synthesized $\mathrm{CuO}$ 
nanoparticles annealed at 200, 400, 600 and $800{ }^{\circ} \mathrm{C}$, respectively.

It is clear from (Fig.1) that intensity of crystalline peaks increases with the increase in temperature indicating the improvement in the crystallinity of samples. Simultaneously, the peaks became narrower as the temperature increase showing the increase in crystallite size and a decrease in the full width at half maximum (FWHM), which indicates a possible change in the grain size of $\mathrm{CuO}{ }^{10,11}$ that can be well seen from

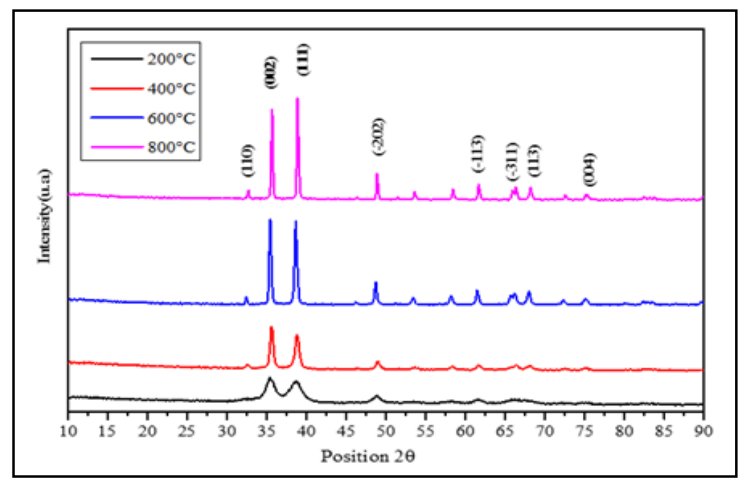

Figure 1. XRD patterns of CuO NPs, at different temperatures

Particle size can be calculated by Debye- Scherrer's formula, $\mathrm{D}=\frac{\mathrm{K} \cdot \lambda}{\beta \cdot \cos \theta}$
(Table.1). Also, this decrease in the (FWHM) values with the growing temperature indicates the enhancement of the crystallite size of the $\mathrm{CuO}$ nanoparticles.

Further, it can also be seen that the major peaks (002) and (111) located at $2 \theta$ values, see (Table.1) correspond to the charascteristic diffraction of monoclinic phase of $\mathrm{CuO}$ (JCPDS NO-65-2309), which concurs Hemanth et al. ${ }^{9}$, XRD results thus confirm synthesis of pure and well crystalline $\mathrm{CuO}$ nanoparticles without any impurity.

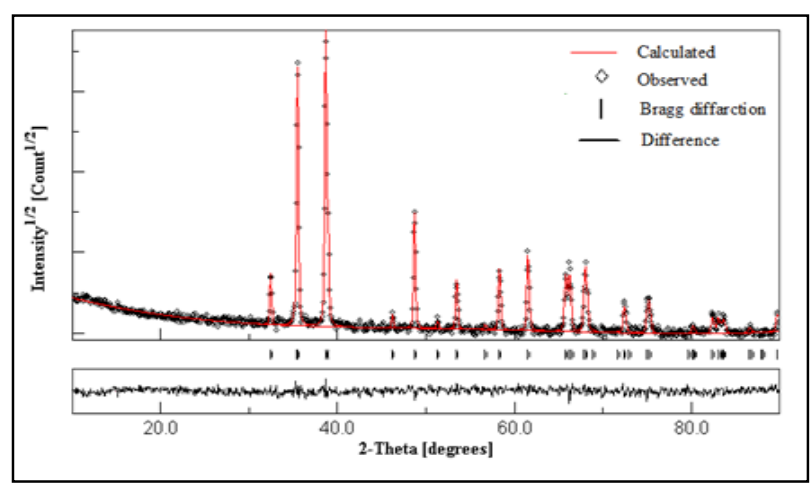

Figure 2. Rietveld refinement patterns of X-ray diffraction data for $\mathrm{CuO}$ NPs annealed at $800^{\circ} \mathrm{C}$.

where $\mathrm{D}$ is the diameter of the crystallite size, $\mathrm{K}$ is the shape factor (the typical value is 0.9 ) 1 is the wavelength of the incident beam, $\beta$ is the broadening of the diffraction line measured in radians at half of its maximum intensity (FWHM), and $\theta$ is the Bragg's angle $^{12}$.

Table 1. FWHM values for the two major peaks and particle size values of annealed CuO samples.

\begin{tabular}{|c|c|c|c|c|c|}
\hline Temperature & $(\mathbf{h k l})$ & $\mathbf{2 \theta}$ & FWHM & Particle size (D) & Particle size from SEM \\
\hline \multirow{2}{*}{$\mathbf{2 0 0}^{\circ} \mathbf{C}$} & 002 & 35.35 & 0.3296 & $5.45 \mathrm{~nm}$ & $9.84 \mathrm{~nm} \pm 1.02$ \\
& 111 & 38.69 & 0.8378 & & \\
\hline \multirow{2}{*}{$\mathbf{4 0 0}^{\circ} \mathbf{C}$} & 002 & 35.62 & 0.3296 & $19.35 \mathrm{~nm}$ & $53.41 \mathrm{~nm} \pm 1.77$ \\
& 111 & 38.81 & 0.4615 & & $131.55 \mathrm{~nm} \pm 3.67$ \\
\hline \multirow{2}{*}{$\mathbf{6 0 0}^{\circ} \mathbf{C}$} & 002 & 35.45 & 0.2637 & $68.07 \mathrm{~nm}$ & \\
\hline \multirow{2}{*}{$\mathbf{8 0 0}^{\circ} \mathbf{C}$} & 111 & 38.63 & 0.3296 & & $188.33 \mathrm{~nm} \pm 2.76$ \\
\hline & 002 & 35.67 & 0.2637 & $172.33 \mathrm{~nm}$ & \\
\hline
\end{tabular}

\section{Morphological studies}

(Fig.3) (a), (b), (c) and (d) exhibit TEM images and size distribution of $\mathrm{CuO}$ nanoparticles annealed at $200^{\circ} \mathrm{C}$, $400^{\circ} \mathrm{C}, 600^{\circ} \mathrm{C}$ and $800^{\circ} \mathrm{C}$ respectively. As observed from (Fig. 2), the $\mathrm{CuO}$ NPs, which were annealed at $200^{\circ} \mathrm{C}$ and $400^{\circ} \mathrm{C}$, are spherical, self-assembled and consisting of particles with unfortunate fusion and aggregation. As the annealing temperature increases to $600^{\circ} \mathrm{C}$ and $800^{\circ} \mathrm{C}$, the sizes of particle are no quite uniform compared with temperatures of $200^{\circ} \mathrm{C}$ and $400^{\circ} \mathrm{C}$ as observed from the TEM results, the particles size are not small anymore and begins to agglomerate and becomes larger than those of lower annealing temperature.

This increase of NP's size with calcination temperature is mainly caused by the agglomeration of particles, which means that at raised temperatures, the atoms possess enough energy (the energy needed for oxide molecules to assemble into crystals). So this energy is communicated to the system by increasing the temperature. Therefore, the molecules are allowed to immigrate towards steady positions and as a consequence, the intensity of peaks and grains size increase.

The histogram of TEM image of CuO NPs confirms that the particle sizes of NPs lie in the range $9-189 \mathrm{~nm}$ (Fig.3), which is in good agreement with those calculated by the Scherrer-Debye formula from the XRD analysis, see (Table.1). The size distribution was obtained by measuring each particle diameter. The random orientation of particles allows for a statistical measure of the size 
distribution to be generated, and the mean value is calculated for each distribution.

The structural parameters for all the calcined samples were refined using Rietveld refinement method, and are given in (Table.2). All the $\mathrm{CuO}$ nanoparticles were crystallized in the monoclinic (tenorite) structure with a space group of $\mathrm{C} 2 / \mathrm{c}$ (No. 15). The observed lattice parameters agree well with the reported data in the standard JCPDS mentioned above. An example of the observed, calculated and the difference refined XRD patterns of samples annealed at $800^{\circ} \mathrm{C}$ are shown in Fig. 3. There is good agreement between the observed and calculated patterns. Further, there is no appreciable change in the lattice parameters of low-temperature calcined samples.

Table 2. Rietveld refined structural parameters for annealed $\mathrm{CuO}$ nanoparticles.

\begin{tabular}{|c|c|c|c|c|c|c|c|}
\hline \multirow{2}{*}{$\begin{array}{c}\text { Annealed } \\
\text { samples }\end{array}$} & \multicolumn{3}{|c|}{ Refined R-Factors } & \multicolumn{4}{c|}{ Lattice parameters } \\
\cline { 2 - 8 } & $\mathrm{R}_{\mathrm{wp}}$ & $\mathrm{R}_{\exp }$ & $\mathrm{R}_{\text {Bragg }}$ & $\mathrm{a}$ & $\mathrm{b}$ & $\mathrm{c}$ & $\beta$ \\
\hline $200^{\circ} \mathrm{C}$ & 13.729 & 3.215 & 8.602 & 4.6869 & 3.4306 & 5.1361 & 99.298 \\
\hline $400^{\circ} \mathrm{C}$ & 10.269 & 4.123 & 7.847 & 4.6808 & 3.432 & 5.1336 & 99.32 \\
\hline $600^{\circ} \mathrm{C}$ & 13.62 & 3.6 & 9.07 & 4.68 & 3.42 & 5.1331 & 99.41 \\
\hline $800^{\circ} \mathrm{C}$ & 11.33 & 4.058 & 5.504 & 4.69 & 3.42 & 5.13 & 99.49 \\
\hline
\end{tabular}
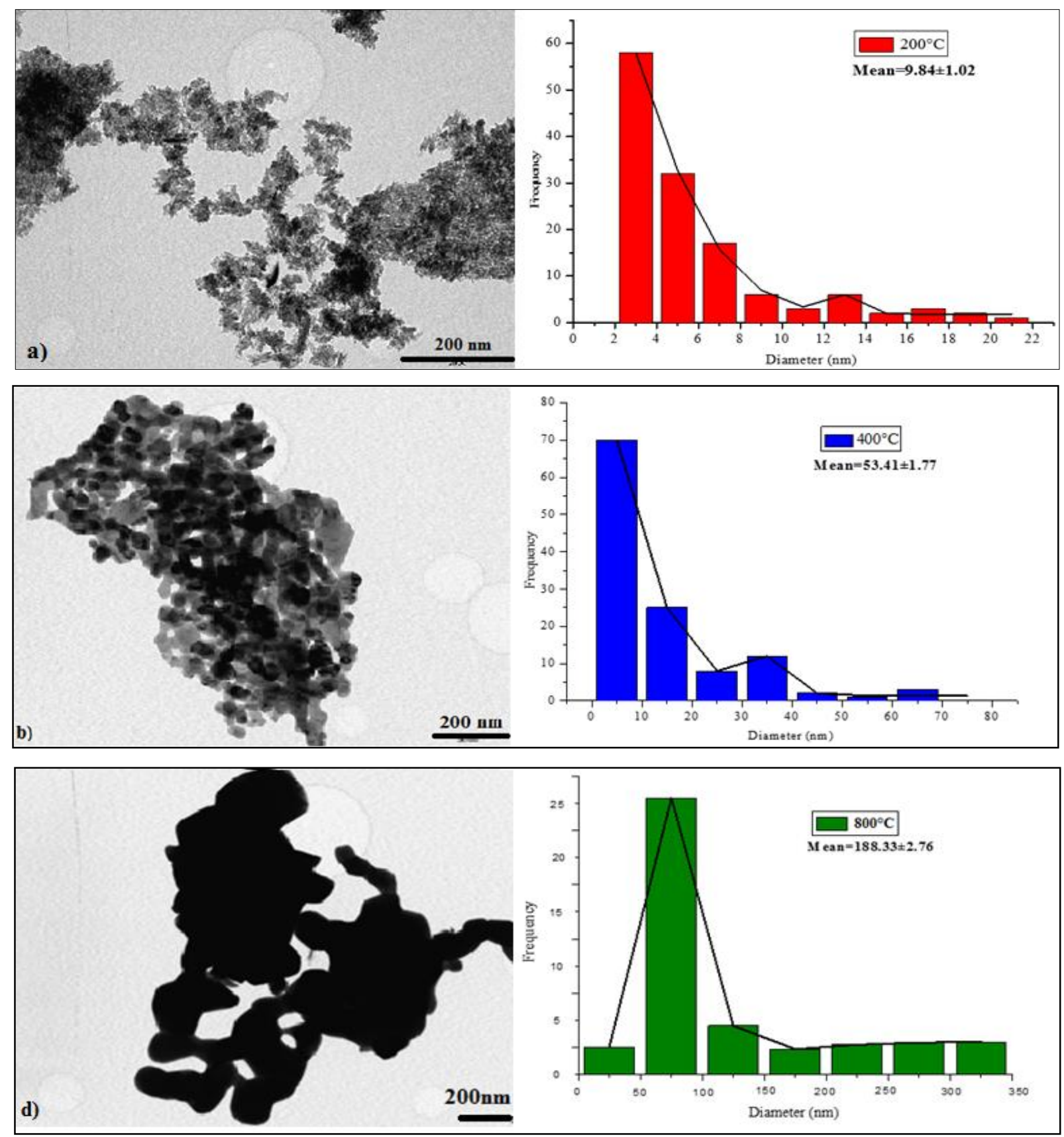


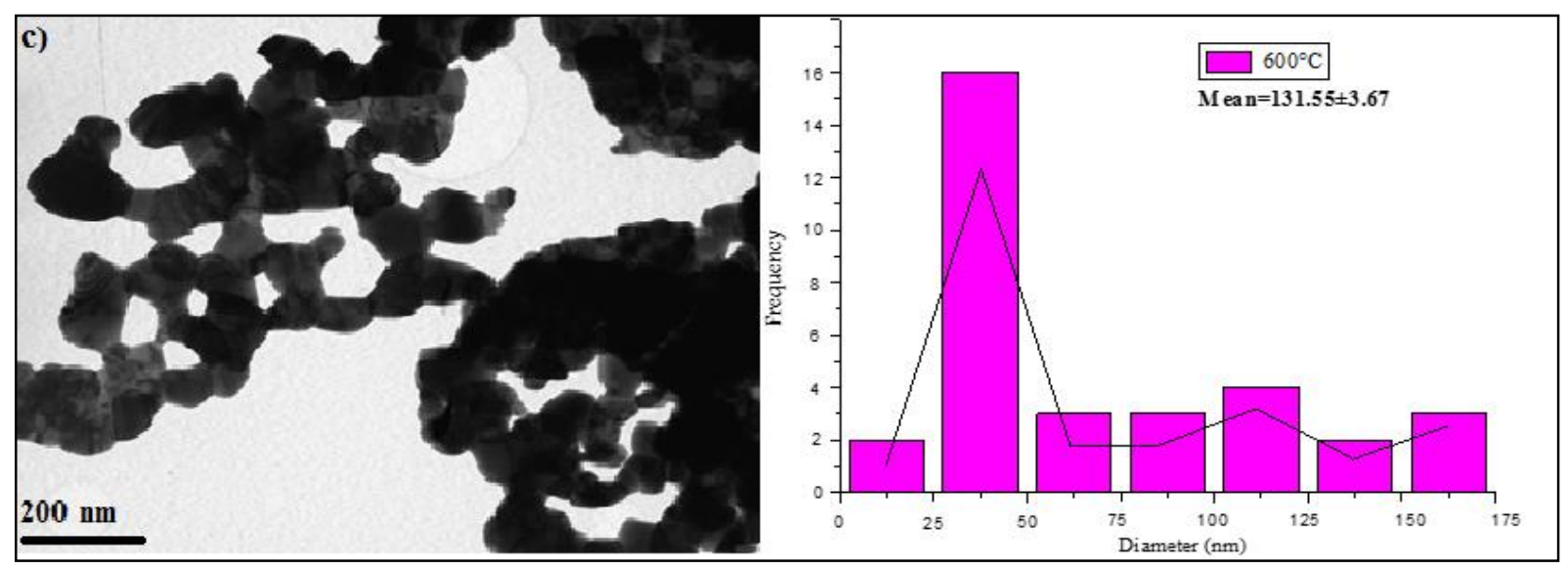

Figure 3. TEM images and particle size distribution of $\mathrm{CuO}$ NPs annealed at a) $200^{\circ} \mathrm{C}$, b) $400^{\circ} \mathrm{C}$, c) $600^{\circ} \mathrm{C}$ and d) $800^{\circ} \mathrm{C}$ respectively.

\section{Optical properties}

The optical properties of $\mathrm{CuO}$ nanoparticle are determined from absorbance measurements from UVVis spectrophotometry in the range of 250-1000 nm. The optical absorption spectrum was used to characterize optical absorption properties of obtained CuO samples ${ }^{13}$.

(Fig.4) shows the UV-Vis absorption spectra of the samples annealed at different temperatures.

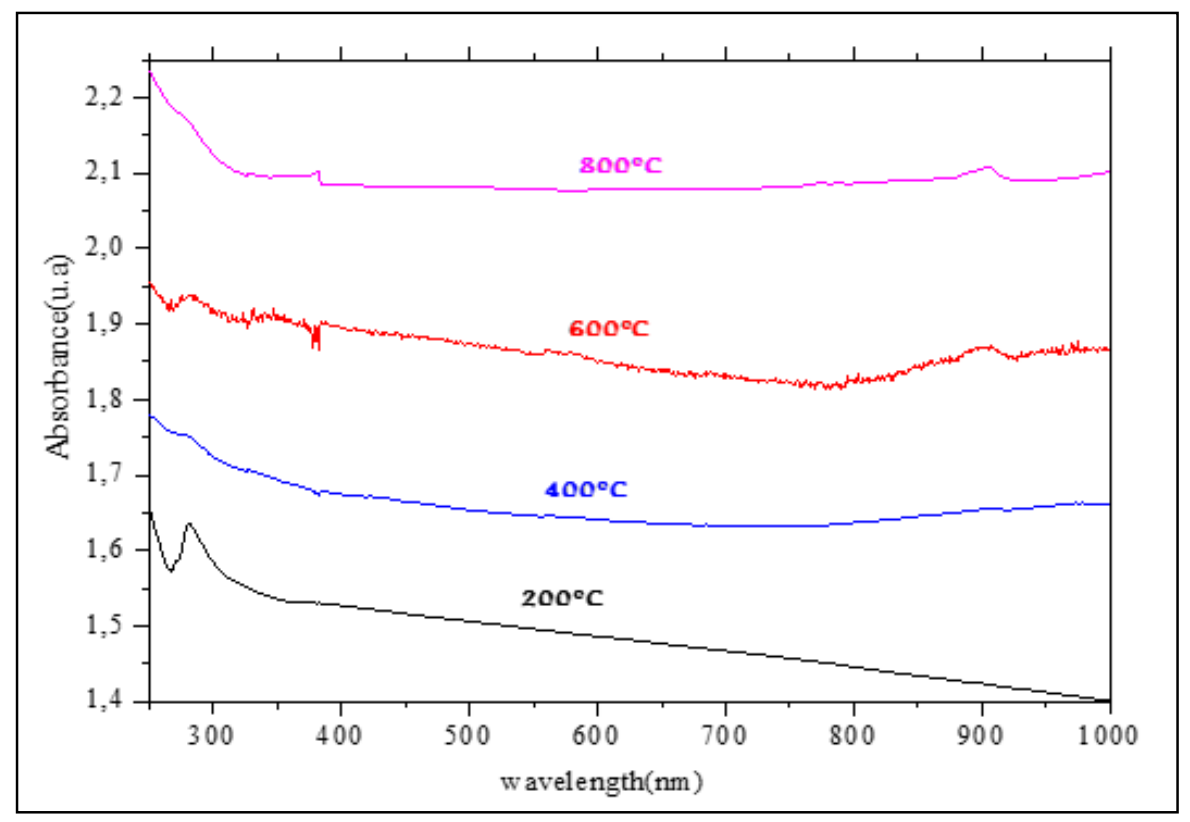

Figure 4. Variation of the absorption coefficient of $\mathrm{CuO}$ nanoparticles as a function of wavelength.

\section{Determination of the Band gap value}

The band gap Eg, can be estimated from the absorption spectra by using the relation (Willardson and Beer):

$(\alpha h v)=A\left(h v-E_{g}\right)^{n}$

Where Eg is the gap energy of the material, $v$ is the frequency of the incident radiation, $h$ is Planck's constant, $\alpha$ is the absorption coefficient in $\mathrm{cm}^{-1}$. A, is a constant related to the material and the matrix element of the transition, and $\mathrm{n}$ is a coefficient depending on the nature of the transition ( $n=1 / 2$ for the direct allowed transition or 2 for an indirect transition).
The absorption coefficient is obtained using the relation

$\alpha \cdot d=\ln \left(\frac{1}{T}\right)$

Where the transmittance $\mathrm{T}$ is calculated from the measured absorbance using the Beer-Lambert law,

$B=-\log _{10} T$

$\mathrm{d}$ represents the path length of the wave in centimeter $(1 \mathrm{~cm})^{13}$.

The linear relationship was obtained by plotting $(\alpha h v)^{2}$ against photon energy (hv). (Fig.5) represents $(\alpha h v)^{2}$ versus hv plots indicating that the absorption edge was due to a direct allowed transition. The line fit to the 
$(\alpha h v)^{2}$ versus hv plot is obtained by fitting a straight line to the linear portion of the curve. The value of optical band gap was determined by extrapolating the values of the coefficient of absorption $\alpha$ to zero. The values of the band were found to be $3.83 \mathrm{eV}, 3.3 \mathrm{eV}, 2.56 \mathrm{eV}$ and 2.25 $\mathrm{eV}$ for the temperatures 200, 400, 600 and $800{ }^{\circ} \mathrm{C}$ respectively, as (Fig.5) shows.

(Fig.6) shows the variation of band gap and grain size with temperature, based on it, it is clear that the band gap decreases with the increase in temperature while the grain size increases.
It has been observed that the band gap was maximum $(3.83 \mathrm{eV})$ when the grain was minimum $(9.84 \mathrm{~nm})$ at $200{ }^{\circ} \mathrm{C}$ while it was minimum $(2.25 \mathrm{eV})$ with a grain size of $205 \mathrm{~nm}$ at $800{ }^{\circ} \mathrm{C}$.

Our results indicate a slight blue shift in the direct band edge as the particle size is reduced. Such a blue shift has also been reported in the literature for $\mathrm{CuO}$ quantum dots ${ }^{14}$ where the blue shift has been attributed to the quantum confinement effects of nanoparticles ${ }^{15}$.

The observed increase in the direct band gap values of $\mathrm{CuO}$ with the decrease in NNPs size is attributed to the quantum confinement effect ${ }^{15}$.

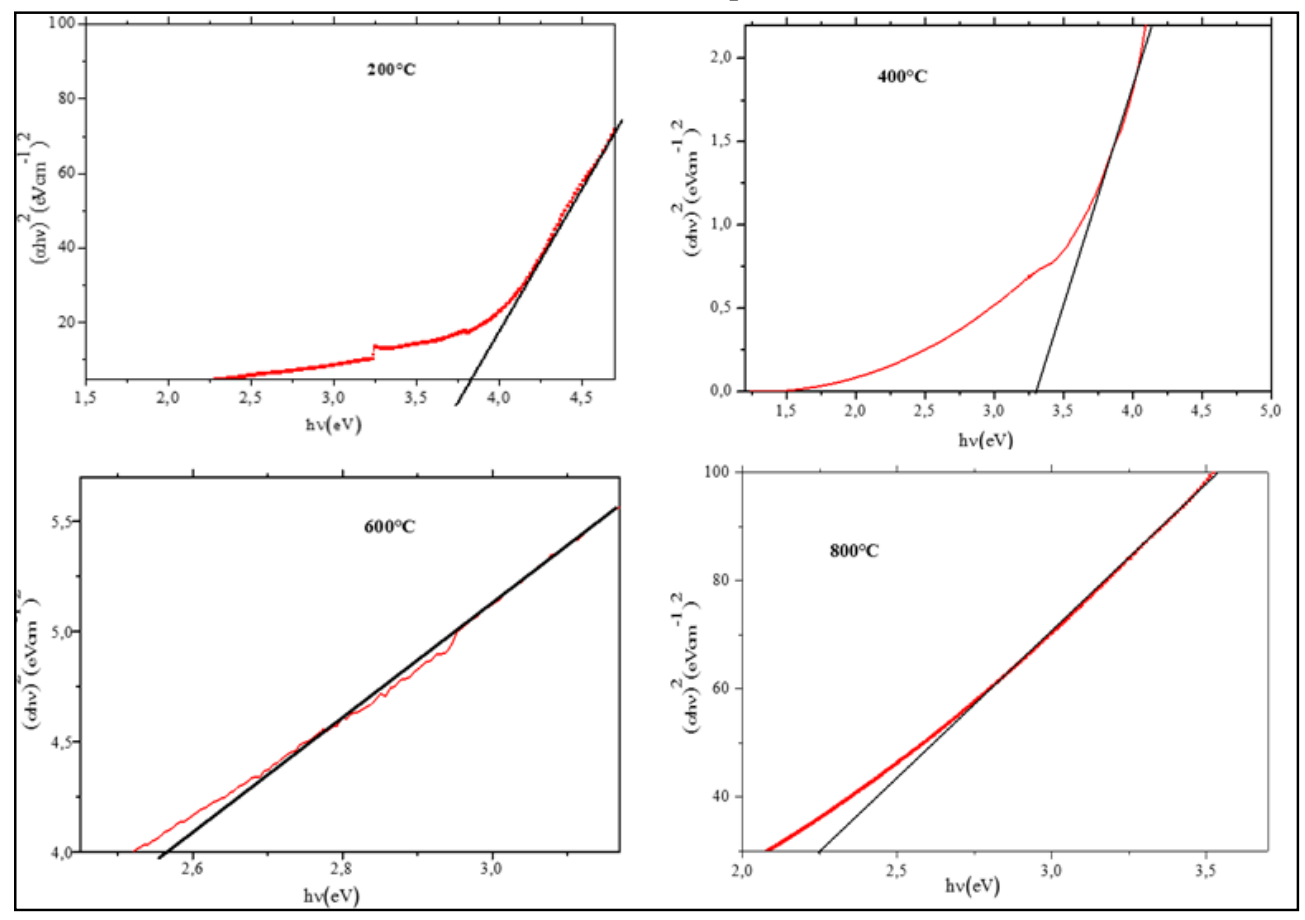

Figure 5. The plot of $\alpha v^{2}$ vs photon energy for $200^{\circ} \mathrm{C}, 400^{\circ} \mathrm{C}, 600^{\circ} \mathrm{C}$ and $800^{\circ} \mathrm{C}$ respectively from

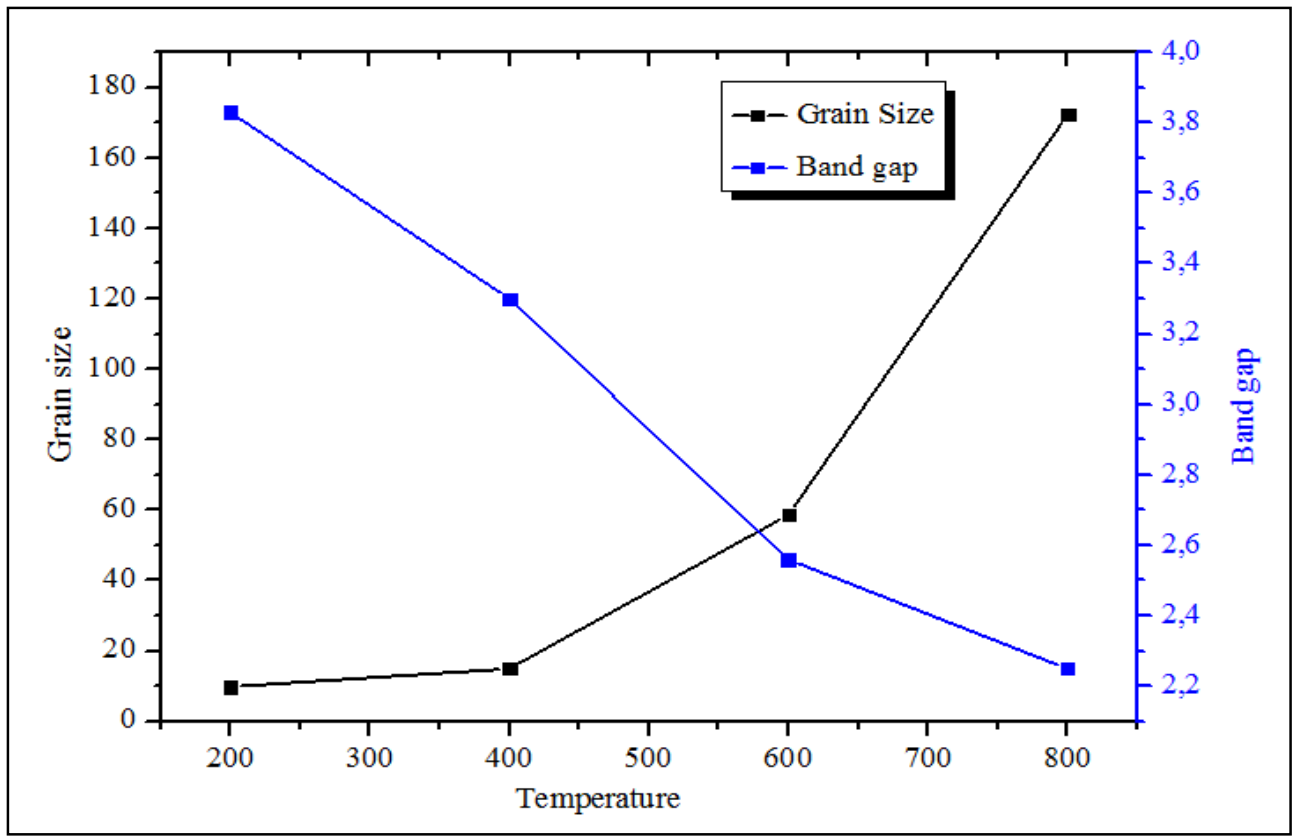

Figure 6. The variation of band gap and grain size with temperature 


\section{Photocatalytic properties}

(Fig.8) shows the linear plots of $\mathrm{C} / \mathrm{C}_{0}$ versus time for the photodegradation of $\mathrm{MB}$ on the two samples of $\mathrm{CuO}$ NPs annealed at $200^{\circ} \mathrm{C}$ and $600^{\circ} \mathrm{C}$ under visible light after 400 min illumination.

The absorption spectra of the samples were recorded by measuring the absorbance at $664 \mathrm{~nm}$ corresponding to the maximum absorption wavelength of MB with UVvisible absorption spectroscopy. The concentration of $\mathrm{MB}$ is proportional to the absorbance of MB according to the Beer-Lambert law, so the degradation efficiency of MB can be calculated by:

$$
R=\frac{C_{0}-C}{C_{0}} * 100
$$

Where $\mathrm{C}_{0}, \mathrm{C}$ are the absorbance and concentration of MB respectively when the reaction time is $t$.

The results show that the photocatalytic activity of the samples depends on the temperature that means in

$$
\log \left(q_{e}-q_{t}\right)=\log q_{e}-\frac{k_{1}}{2.303} t
$$

Where, qe and qt are the amount of the dye adsorbed on the adsorbent $(\mathrm{mol} / \mathrm{g})$ at equilibrium and time $\mathrm{t}, \mathrm{k}_{1}$ is the rate constant (min-1) representing the pseudo-firstorder kinetics, and $\mathrm{k}_{2}$ is the adsorption of pseudo-secondorder rate constant (mol/g.min). The pseudo-second- another term, the NPs size. It was found that almost $60.75 \%, 68.3 \%, 75.89 \%$, and $80.03 \%$ of methylene blue dye were degraded after $540 \mathrm{~min}$ UV illumination for samples annealed at $200,400,600,800^{\circ} \mathrm{C}$ respectively. It is obvious that the photocatalytic activity of $\mathrm{CuO}$ samples annealed at higher temperature $\left(800^{\circ} \mathrm{C}\right)$ have better photocatalytic activity, and this could be associated with their big particles size, so naturally exhibiting large specific surface sites which have been exposed for dye molecules, compared to those annealed at lower temperature, small particles size, smaller surface area.

For the investigation of the possible mechanism of adsorption, the kinetics of the photodegradation reaction was studied using the Lagergren pseudo-first order kinetics model as expressed ${ }^{16}$ :

$\& \frac{t}{q_{t}}=\frac{1}{K_{2} q_{e}^{2}}+\frac{t}{q_{e}}$

order adsorption kinetic plot is shown in (Fig.7), which shows that it follows a pseudo-second-order reaction and which indicates hat the equilibrium was established between MB dye and $\mathrm{CuO}$ NPs within $10 \mathrm{~min}$.

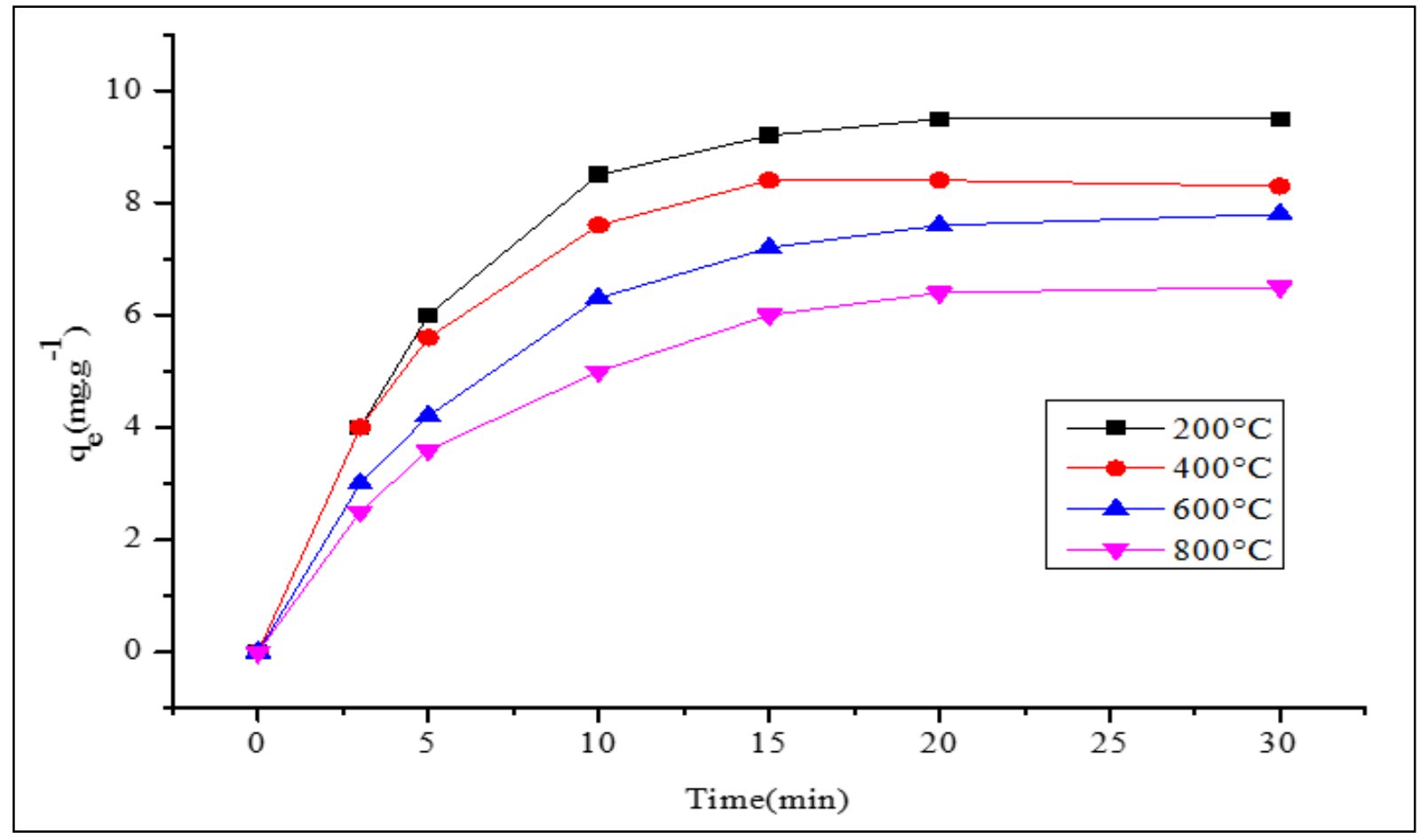

Figure 7. Kinetics of methylene blue sorption on annealed $\mathrm{CuO}$ NPs. 


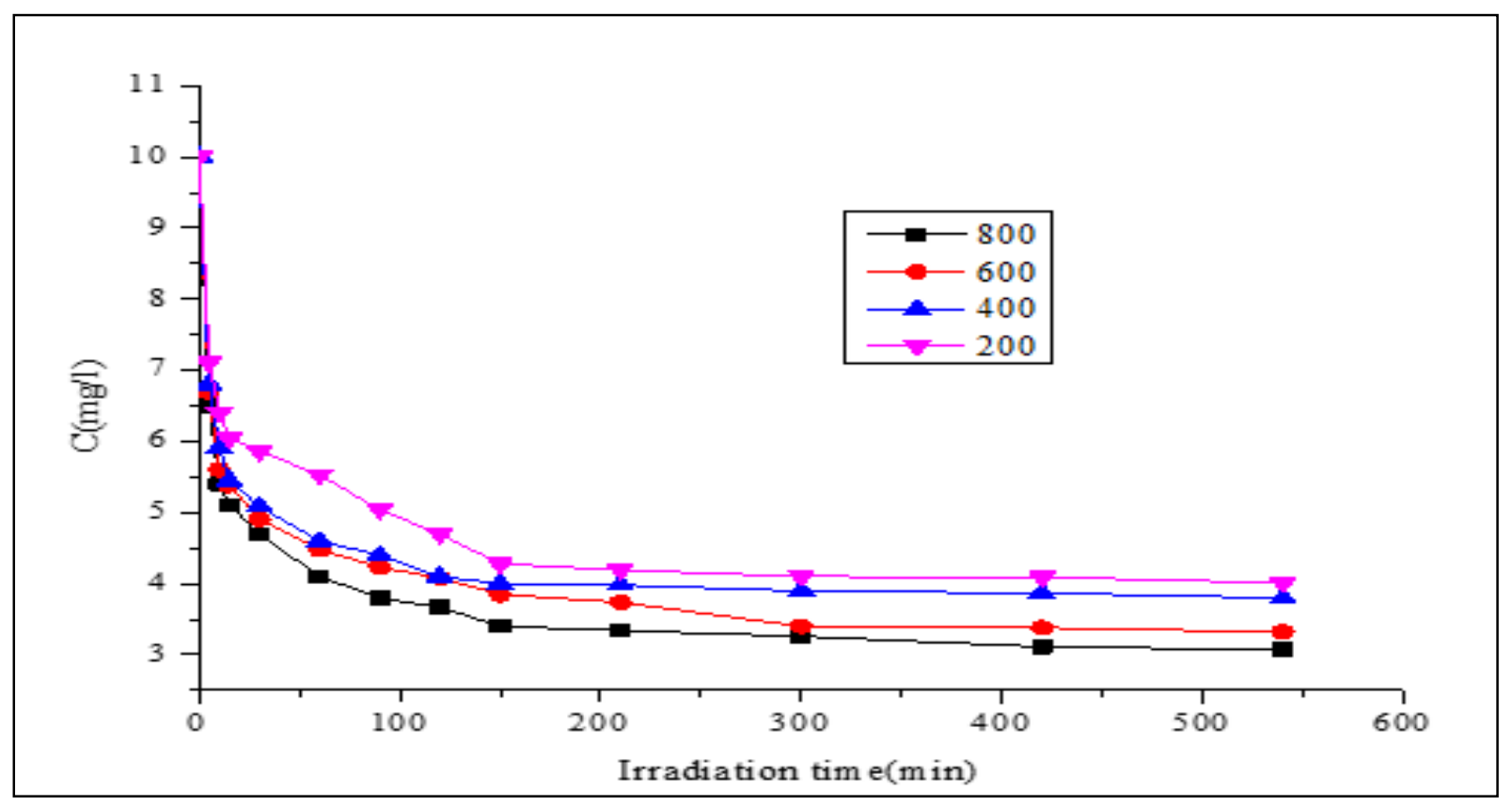

Figure 8. Photodegradation of MB on CuO NPs annealed at $200,400,600$ and $800^{\circ} \mathrm{C}$.

\section{Conclusion}

$\mathrm{CuO}$ NPs were synthesized by sol-gel combustion method. X-ray diffraction measurements revealed that the crystallite size, lattice parameters and crystallinity of $\mathrm{CuO}$ nanoparticles have enhanced with the elevation in annealing temperature. TEM analysis indicated that the grain size evaluates proportionally with the evolution of temperature, which was well confirmed by XRD results. UV-Visible analysis indicated also that by reducing the NP's size the band gap raise to the higher values which was well confirmed in other way by Jin Zhong Zhang that when reducing in NP's size, the density of states becomes more quantized ${ }^{17}$, (effect of reducing temperature, lack of excitement for the electron), so increasing in the band gap. On the other hand, the optical band gap of the $\mathrm{CuO}$ nanoparticles was found to be higher as compared to a bulk value indicating a blue shift of band gap, which has been attributed to the quantum confinement effect. Photocatalytic degradation of methylene blue was carried out the as-synthetized nanoparticles at four different annealing temperatures under visible light irradiation. The results have shown that the photocatalytic activity of the samples depended on the annealing temperature that they endured, so in our experiment, the photodegradation efficiency was promoted by increasing the annealing temperature so by augmenting the $\mathrm{CuO}$ NPs size.

\section{Acknowledgement}

The authors would like to acknowledge the CNRST, Rabat, Morocco for the experimental measurements: TEM, XRD and UV-vis analysis, and a special thank is dedicated to the LCPG laboratory, Department of Chemistry, Faculty of Sciences, Mohammed V University, Rabat, Morocco, for the photodegradation measurements.

\section{References}

1- Serena A. Corr, Nanoscience: Metal oxide nanoparticles; ed. by P. O'Brien; RSC, UK, 2012, pp. 180-207.

2- R. Katwal, H. Kaur, G. Sharma, M. Naushad, D. Pathania, Electrochemical synthesized copper oxide nanoparticles for enhanced photocatalytic and antimicrobial activity, J. Ind. Eng. Chem. 2015, 2566, $1-12$.

3- T. Ishihara, M.Higuchi, T.Takagi, M. Ito, H. Nishiguchi, T.Takita, Preparation of $\mathrm{CuO}$ thin films on porous $\mathrm{BaTiO}_{3}$ by self-assembled multilayer film formation and application as a $\mathrm{CO}_{2}$ sensor, $\mathrm{J}$ mater Chem., 1998, 8, 2037-2042.

4- C. Xu, G. P. Rangaiah, X. S. Zhao, Photocatalytic Degradation of Methylene Blue by Titanium Dioxide: Experimental and Modeling Study, Ind. Eng. Chem. Res., 2014, 53, 14641-14649.

5- W, El rouby, A. al-ghamdi, M. abdel-wahab, A. Jilani, Sunlight-enhanced catalytic degradation over $\mathrm{Ag}-$ $\mathrm{CuO}$ nanoparticles thin films prepared by $\mathrm{DC} / \mathrm{RF}$ sputtering technique, Bull. Mater. Sci. 2018, 41:58, 111.

6- A. Tadjarodi, O. Akhavan, K. Bijanzad, Photocatalytic activity of $\mathrm{CuO}$ nanoparticles incorporated in mesoporous structure prepared from bis (2-aminonicotinato) copper (II) microflakes, Transactions of nonferrous metals. Society of China, 2015, 25, 3634-3642.

7- A. Zoolfakar, N. Abu Bazar, N. Khairir, Y. Husaini, M. Abu Talip, R. Abdul Rani, M. Rusop, Synthesis and Enhanced Photocatalytic Property of $\mathrm{CuO}$ Nanostructure via Dip Coating Method, IEEE. 2017, 978, 30-33.

8- P. Chand, A. Gaur, A. Kumar, Study of $\mathrm{CuO}$ nanoparticles synthesized by sol-gel method, AIP Conference Proceedings, 2011, 211, 1393. 
9- R. Hemanth, M. Sekar, B. Suresha, Effects of fibers and fillers on mechanical properties of thermoplastic composites, Indian Journal of Advances in Chemical Science, 2014, 2, 28-35.

10-C.C. Vidyasagar, Y.A. Naik, T.G. Venkatesh, R. Viswanatha, Solid-State Synthesis and Effect of Temperature on Optical Properties of $\mathrm{CuO}$ Nanoparticles, Powder Technol. 2011, 214, 337-343.

11-P. Mallick, S. Sahu, Structure, Microstructure and Optical Absorption Analysis of $\mathrm{CuO}$ Nanoparticles Synthesized by Sol-Gel Route, Nanosci \& Nanotechnol. 2012, 2, 71-74.

12-G. Varughese, V. Rini, S.P. Suraj, K.T. Usha, Characterisation and optical studies of copper oxide Nanostructures doped with lanthanum ions, ADV. MAT. SCI, 2014, 4 (42), 49-60.

13-N. Zayyoun, B. Jaber, L. Laânab, E. Ntsoenzok, R. Bekkari, Effect of solvent on the morphological and optical properties of $\mathrm{CuO}$ nanoparticles prepared by simple sol-gel process, J. Mater. Environ. Sci., 2016, 7(5), 1791-1797.

14-K. Borgohain, S. Mahamuni, Formation of singlephase $\mathrm{CuO}$ quantum particles, J. Mater. Res. 2002, 17, 1220-1223.

15-L.C. Bourne, P.Y. Yu, A. Zettl, M.L. Cohen, Highpressure electrical conductivity measurements in the copper oxides, Phys. Rev. B., 1989, 40, 10973-10976.

16-H. Bouyarmane, S. Saoiabi, I, El Hanbali1, M. El Karbane, A. Rami, S. Masse, A. Laghzizil, T. Coradin, Porous hydroxyapatite-TiO2 nanocomposites from natural phosphates and their decolorization properties, Eur. Phys. J. Special Topics, 2015, 224, 1863-1871.

17-J. Z. Zhang, Optical properties and spectroscopy of nanomaterials; ed. by World Scientific Publishing Co. Pte. Ltd 2009, pp. 229-299. 